SAHUARUS

Revista Electrónica de Matemáticas,

Departamento de Matemáticas, Universidad de Sonora,

Vol. 1, No. 2, Septiembre, 2016, pp. 1-18.

\title{
Caos: ¿un orden para el desorden?
}

\author{
Víctor F. Breña Medina \\ Centro de Ciencias Matemáticas, UNAM Campus Morelia \\ e-mail: victorb@matmor.unam.mx
}

Resumen

\begin{abstract}
En este artículo se describen las características esenciales que determinan un comportamiento caótico en los sistemas dinámicos. Con este fin, se reproduce el análisis de la Ecuación Logística. También, se muestran los conceptos de dimensión fractal y autosimilitud por medio del Conjunto de Mandelbrot. La sensibilidad a condiciones iniciales es ejemplificada por el Sistema de Lorenz. Finalmente, se exponen los ingredientes necesarios de la ruta RuelleTakens-Newhouse al caos en el sistema de reacción-difusión Barrio-Varea-Aragón-Maini.
\end{abstract}

\section{Introducción}

El caos es una propiedad de la naturaleza. Con el fin de entender este fenómeno, mostraremos las características que componen la teoría del caos.

Primero entendamos las reglas básicas del juego. Un sistema dinámico consiste en: (a) un conjunto de variables dependientes llamadas estados, (b) un conjunto de variables independientes conocidas como parámetros de evolución, (c) un conjunto de parámetros y (d) un conjunto de reglas. Los estados corresponden a variables específicas de un fenómeno, por ejemplo: el número de individuos o densidad de los mismos en una población de animales o de una familia de proteínas en una célula. Este conjunto cambia respecto a los parámetros de evolución, los cuales son usualmente el tiempo o la posición. Propiedades como la temperatura, tasa de natalidad o el tamaño de una región donde ocurre una interacción bioquímica son ejemplos de parámetros; estos caracterizan las propiedades que influyen en los estados del fenómeno en estudio pero que, sin embargo, no varían con respecto a los parámetros de evolución. Las reglas que capturan todos estos ingredientes se traducen en términos de una relación de correspondencia, la cual usualmente es de naturaleza no lineal. De esta manera, los sistemas dinámicos pueden clasificarse en dos grandes grupos, escritos en forma general,

- Discretos.

Ecuación en diferencias: $\mathbf{u}_{t+1}=\mathbf{f}\left(\mathbf{u}_{t} ; \boldsymbol{\mu}\right)$, donde $t \in \mathbb{N}_{0}, \mathbf{u} \in \mathbb{R}^{n}$ y $\boldsymbol{\mu} \in \mathbb{R}^{p}$. Aunque solamente estamos interesados en variables de evolución que aumentan positivamente (futuro), las ecuaciones en diferencias pueden extenderse también para tiempos negativos (pasado); de esta manera, cuando la relación de correspondencia $\mathbf{f}$ es invertible, $t \in \mathbb{Z}$. Cuando estos estados determinan una propiedad específica de una celda a partir de un conjunto de reglas en las celdas vecinas en una red regular, el sistema dinámico es conocido como autómata celular; véanse, para mayores detalles, (Pickover, 2009; Schiff, 2008). Este es un ejemplo de los sistema dinámicos discretos extendidos, donde $\mathbf{u} \in \mathbb{R}^{\mathbb{Z}}$. 
- Continuos.

Ecuación diferencial ordinaria: $\dot{\mathbf{u}}=\mathbf{f}(t, \mathbf{u} ; \boldsymbol{\mu})$, donde $t \in \mathbb{R}, \mathbf{u} \in \mathbb{R}^{n}$ y $\boldsymbol{\mu} \in \mathbb{R}^{p}$. Cuando la posición es también un parámetro de evolución, el sistema dinámico toma la forma de una ecuación diferencial parcial, por ejemplo $\mathbf{u}_{t}=\mathbf{f}\left(t, \mathbf{x}, \mathbf{u}, \mathrm{Du}, \ldots, \mathrm{D}^{m} \mathbf{u} ; \boldsymbol{\mu}\right)$, donde $\mathbf{u} \in \mathbb{R}^{n},(t, \mathbf{x}) \in \mathbb{R} \times \mathbb{R}^{d}$ y $\boldsymbol{\mu} \in \mathbb{R}^{p}$; aquí $\mathrm{D}^{m} \mathbf{u}$ representa la $m$-ésima derivada del estado $\mathbf{u}$ respecto a todas las entradas del parámetro de evolución $\mathbf{x}$ y $\mathbf{u}_{t}$ la derivada parcial respecto a $t$.

Entenderemos que la dinámica de un sistema está dada por las características de los estados al variar los parámetros de evolución, para distintos valores de los parámetros que caracterizan un fenómeno determinado; por ejemplo: el crecimiento de una población de ballenas según algún ciclo de migración (ecuaciones en diferencias), el proceso de transporte de una hormona en un conjunto de células en la epidermis de una planta (autómatas celulares), un brote epidémico en una ciudad (ecuaciones diferenciales ordinarias) o el proceso de turgencia debido a la diferenciación de una población de células (ecuaciones diferenciales parciales). En este espíritu, no es sorprendente que los sistemas dinámicos es una de las teorías más ricas en las matemáticas. Es decir, esta teoría captura un amplio espectro de herramientas, técnicas y teorías de otras ramas del conocimiento matemático, desde la topología hasta la geometría algebraica y todas sus injerencias en las llamadas matemáticas aplicadas, por ejemplo.

A lo largo de este texto, cuando abordemos ejemplos de sistemas continuos, utilizaremos la noción de espacio fase. Este es un espacio donde todos los posibles estados de un sistema dinámico corresponden a un punto único. De esta manera, para un conjunto de parámetros fijos, la evolución de un estado inicial es representada por medio de una curva en el espacio fase, la cual es comúnmente denominada órbita. Aunque este concepto quedará definido de manera precisa más adelante, con las ideas expuestas hasta este punto, estamos en condiciones de comenzar nuestro viaje hacia la teoría del caos.

En su ensayo A Philosophical Essay on Probabilities, Pierre Simon Laplace (véase Laplace, 1902) sugirió que, si un espectador fuese capaz de conocer todas las fuerzas que determinan el movimiento en la naturaleza, así como las posiciones de las partículas que lo componen, dicho individuo podría determinar completamente el futuro y pasado del universo. En otras palabras, Laplace propuso que solamente las condiciones iniciales del universo bastarían para predecir todo evento posterior al inicio del mismo. Posiblemente esta sea una descripción precisa de lo que conocemos como determinismo. Sin embargo, aunque la imagen de esta idea se antoja maravillosa, la naturaleza siempre nos juega malas (y buenas) bromas cuando creemos que la hemos entendido cabalmente. Existen ejemplos de fenómenos donde predecir un evento en su totalidad es una tarea literalmente imposible. Por ejemplo, a principios del siglo XX, Henri Poincaré escribió en su tratado Les Méthodes Nouvelles de la Mécanique Celeste (véase Poincaré, 1899) que, en algunos sistemas astronómicos, el más pequeño error de medición podría propagarse de tal manera que haría imposible la predicción de la dinámica de un fenómeno astronómico. El ejemplo clásico por excelencia es conocido como el problema de los tres cuerpos, el cual consiste en tres cuerpos celestes cuya dinámica es gobernada por la física Newtoniana, por ejemplo: la Tierra, el Sol y la Luna. Poincaré demostró que la más pequeña diferencia en las mediciones iniciales, conduciría a resultados muy distintos. Esta 
idea está relacionada cercanamente con el concepto de inestabilidad, la cual determina cuando los estados de un sistema no persisten ante ciertas perturbaciones. Como veremos en los ejemplos que discutiremos aquí, hay sistemas que siguen un conjunto de reglas deterministas, pero en los cuales, las variaciones que los estados sufren al evolucionar en las variables independientes resultan imposibles de predecir.

En estos términos, es posible definir al caos como la falta de "orden" de un sistema dinámico no lineal que, sin embargo, obedece a un conjunto de reglas deterministas.

\section{Paso a pasito rumbo al caos}

Para comenzar a dar forma a la conexión entre la falta de orden y el determinismo, veremos un ejemplo donde se formulan hipótesis muy sencillas pero que aborda un fenómeno complejo. Al final de esta sección, estarán sentadas las bases que nos permitirán entender una de las características fundamentales de algunos sistemas que exhiben un comportamiento caótico: los atractores extraños.

\subsection{La discreción del caos}

El modelo cuya dinámica observaremos es conocido como el modelo de crecimiento logístico discreto o simplemente ecuación logística. Primero, deduciremos las reglas del modelo y, posteriormente, veremos las implicaciones que éstas tienen.

Imaginemos que queremos predecir el futuro de una población de individuos cuya cantidad limitada de alimento en una determinada región de tamaño finito, por ejemplo, una población de cerdos en una isla. Tomaremos el ejemplo más simple, es decir, una idealización: supondremos que los cerdos nacen, crecen, se reproducen y mueren en la isla. En este caso queremos dar respuesta a preguntas tales como: ¿es posible determinar condiciones para que la población sobreviva y así evitar la extinción? O bien, dado que la cantidad de alimento es determinante para la existencia de cualquier población, ¿qué tanto y cómo influye en la evolución de ésta en el transcurso del tiempo? También podríamos dar respuesta a preguntas como: ¿qué tanto influye la rapidez con la que se reproducen los individuos de la población al transcurrir el tiempo? y ¿será cierto que solamente conociendo la población inicial y la cantidad de alimento disponible en la isla podremos predecir el futuro de la misma?

Vayamos dando nombre a los ingredientes del modelo. Pensemos que $t$ es el tiempo en el que contamos el número de individuos de la población. Por ejemplo, el tiempo de incubación de los cerdos es de aproximadamente tres meses, entonces $t$ tiene unidades trimestrales de tiempo. De esta manera, la colección de cantidades $\left\{N_{0}, N_{1}, \ldots\right\}=\left\{N_{t}\right\}_{t \in \mathbb{N}_{0}}$ representa el número de individuos en cada tiempo $t$. Llegados a este punto, imponemos nuestra primera condición: $N_{t} \geq 0$ para todo tiempo. Dado que nos interesa conocer el número de individuos en el trimestre $t+1$, es decir, nos interesa conocer el valor de $N_{t+1}$, el cuál llamaremos el futuro inmediato de la población, tenemos que deducir una regla de correspondencia entre el número de individuos en la población al tiempo $t$, el presente, y en el futuro inmediato. Debido a que el alimento es limitado, cuando el número de individuos es menor que el número 
que puede sostener su entorno, la población crece, es decir $N_{t}<N_{t+1}$; en caso contrario, $N_{t}>N_{t+1}$, debido a la escasez de espacio y alimento.

Existen varias posibilidades para capturar las condiciones arriba descritas. Aquí consideraremos el caso más sencillo, propuesto por Robert May (véase May, 1976). Según este autor, la razón de cambio de la población está dada por la diferencia entre la cantidad de individuos en el futuro inmediato y los individuos en el presente por unidad de tiempo, es decir: $N_{t+1}-N_{t}$. De esta manera, esta diferencia es favorecida de manera directamente proporcional al número de individuos al tiempo $t$. De igual manera, el número de individuos que los recursos (alimento y espacio) pueden sostener desfavorecen esta razón de crecimiento. Para plantear esto de manera general, pensemos que $\alpha$ es un parámetro positivo que indica la razón de crecimiento de la población y, por otro lado, $\kappa$ representa la cantidad de alimento en la isla. Este parámetro es conocido como la capacidad de carga. Por lo tanto, la razón de cambio de la población de individuos en el intervalo de tiempo $[t, t+1]$ es directamente proporcional a la diferencia entre el número de individuos que nacieron y el total que pueden ser alimentados en la isla. De esta forma obtenemos la siguiente ecuación:

$$
N_{t+1}-N_{t}=\alpha N_{t}-\alpha N_{t} \frac{N_{t}}{\kappa}
$$

El término derecho de esta ecuación determina que, para valores de $N_{t}$ pequeños, la diferencia en el lado izquierdo es positiva; en otras palabras, cuando el número de individuos en el presente, $N_{t}$, es pequeño, la población crecerá en el futuro inmediato $t+1$. Lo contrario ocurre cuando $N_{t}$ es un valor cercano a $\mathcal{N}^{*}=(1+\alpha) \kappa / \alpha$. El valor de la población $\mathcal{N}^{*}$ indica la cantidad de individuos que permanence constante para cualquier tiempo $t$; es decir: $N_{t}=N_{t+1}$ para cualquier valor de $t$. Observemos que entre mayor sea el valor de $\kappa$, mayor es $\mathcal{N}^{*}$. Lo mismo sucede cuando $\alpha$ decrece. Es decir, entre mayor sea el alimento disponible en la isla o la razón de crecimiento sea menor, el número de individuos determinada por $\mathcal{N}^{*}$ es mayor.

Ahora, para simplificar el análisis, definamos el parámetro Malthusiano $r \equiv 1+\alpha$ y la variable relativa $x_{t} \equiv N_{t} / \mathcal{N}^{*}$. Sustituyendo esta nueva variable en (1), obtenemos la ecuación logística:

$$
x_{t+1}=f\left(x_{t} ; r\right), \quad f(x ; r) \equiv r x(1-x) .
$$

Debido a que $0 \leq x_{t} \leq 1$ para todo tiempo $t$, los valores admisibles del parámetro $r$ son aquellos tales que $0 \leq r \leq 4$; es decir, a partir de (2), se satisface que $0 \leq x_{t+1} \leq 1$ cuando $r=4$ y $0 \leq x_{t+1}<1$ si $r<4$, para cualquier valor de $t$.

La ecuación (2), que determina la dinámica de la población relativa $x_{t}$, indica que $x_{0}$ al tiempo $t=0$ determina la población relativa $x_{1}$ al tiempo $t=1$, de tal manera que $x_{1}=f\left(x_{0} ; r\right)$; cuando $t=2$ tenemos que

$$
x_{2}=f\left(x_{1} ; r\right)=f\left(f\left(x_{0} ; r\right) ; r\right)=f^{2}\left(x_{0} ; r\right) .
$$

Es decir, para conocer la población relativa $x_{2}$, iteramos dos veces la función $f$. Procediendo 
de esta forma, la población relativa al tiempo $t=n$ está dada por

$$
x_{n}=\underbrace{(f \circ \cdots \circ f)}_{n \text {-veces }}\left(x_{0} ; r\right)=f^{n}\left(x_{0} ; r\right) ;
$$

de esta forma, la población relativa $x_{n}$ al tiempo $t=n$, depende de la población relativa inicial y del parámetro Malthusiano solamente. Más aún, de esta manera, la colección de puntos $\left\{x_{0}, x_{1}, \ldots\right\}$ es dada por la relación (3). Este conjunto determina una órbita de la ecuación (2). En otras palabras, cada elemento de la órbita de una población relativa inicial dada depende del parámetro Malthusiano por medio de (3).

Existen valores de $x_{t}$ tales que permanecen constantes al variar el tiempo $t$; éstos son identificados si se satisface que $x_{t+1}=x_{t}$ para cualquier valor de $t$. Esta condición es equivalente a buscar las raíces $x^{*}$ de la ecuación $f\left(x^{*} ; r\right)-x^{*}=0$, las cuales se conocen como puntos fijos. La naturaleza de estos puntos está determinada por el parámetro $r$. En otras palabras, los puntos fijos pueden ser atractores o repulsores; es decir, atrapan o repelen una órbita $\left\{x_{t}\right\}_{t \in \mathbb{N}_{0}}$, la cual puede también entenderse como la evolución temporal de una población relativa inicial. Esta clasificación es determinada por la derivada de la función $f$ en el punto fijo de la siguiente manera:

i. $x^{*}$ es un atractor, si $\left|f^{\prime}\left(x^{*} ; r\right)\right|<1$ y

ii. si $\left|f^{\prime}\left(x^{*} ; r\right)\right|>1$, se dice que $x^{*}$ es un repulsor.

En los casos donde $\left|f^{\prime}\left(x^{*} ; r\right)\right|=1$ conforme cambia el valor del parámetro $r$, ocurre un cambio dramático en la dinámica del sistema; este cambio dramático es conocido como bifurcación. Notemos que, en particular, la condición $f^{\prime}\left(x^{*} ; r\right)=-1$ indica que la recta tangente a $f$ en el punto $x^{*}$ es perpendicular a la recta identidad. Debido a que los puntos fijos son determinados por la intersección entre la recta identidad y la gráfica de la relación de correspondencia, éstos siempre yacen en la identidad y en $f$ cuando existen. Ahora, cuando esta propiedad de perpendicularidad se satisface, se dice que el punto fijo es periódico, lo cual quiere decir que $x_{t+p}=x_{t}$ para todo tiempo, donde $p$ es el período de la órbita. Como consecuencia, se obtiene que la $p$-ésima iteración de $f$ es igual a la identidad, i.e. se satisface que $x=f^{p}(x ; r)$ (véase Robinson, 1999, para una discusión detallada).

Llegados a este punto, tenemos los ingredientes necesarios para regresar a la ecuación logística. Los puntos fijos de (2) son

$$
x_{0}^{*}=0 \quad \mathrm{y} \quad x_{r}^{*}=1-\frac{1}{r} .
$$

Esto indica que si $r \leq 1$, el origen es el único punto fijo y si $1<r \leq 4$, también $x_{r}^{*}$ es punto fijo. Si $x_{0}^{*}$ es un atractor, entonces, dada cualquier población relativa inicial, la órbita convergerá al origen conforme el tiempo crece; esto significa que la población disminuirá al transcurrir el tiempo hasta llegar a la extinción. Por el contrario, si $x_{0}^{*}$ es repulsor y el punto fijo $x_{r}^{*}$ es atractor, entonces la población alcanzará un valor determinado por el parámetro Malthusiano al transcurrir el tiempo y, por tanto, no se extingue.

Debido a que $\alpha>0$, entonces $r>1 \mathrm{y}$, por lo tanto, el origen es repulsor para cualquier valor de $r$ admisible; esto sugiere que $x_{r}^{*}$ podría ser el único atractor. En este contexto, como 
veremos a continuación, la dinámica que determina la supervivencia es diversa. Con este fin, analizaremos la naturaleza de los puntos fijos para todos los valores del parámetro $r$ tales que $1<r \leq 4$; este intervalo recibe el nombre de intervalo admisible.

En el intervalo admisible, los puntos fijos están dados por (4). Al calcular la primer derivada de $f$ en cada punto fijo, notamos que $f^{\prime}\left(x_{0}^{*} ; r\right)=r$ y $f^{\prime}\left(x_{r}^{*} ; r\right)=2-r$. Como primer consecuencia, cualquier órbita de (2) diverge de $x_{0}^{*}$ y convergen en $x_{r}^{*}$ en el límite, si $\left|f^{\prime}\left(x_{r}^{*} ; r\right)\right|<1$. De esta manera, como veremos a continuación, deducimos ciertas condiciones específicas en el parámetro $r$.

Observemos que $f^{\prime}\left(x_{r}^{*} ; r\right)=1$ cuando $r=1$ y $f^{\prime}\left(x_{r}^{*} ; r\right)=-1$ para $r=3$. La primera de estas condiciones no se encuentra en el intervalo admisible. Por el contrario, la segunda de estas condiciones es plausible. Esto significa que el sistema (2) sufre una bifurcación de doblamiento de período cuando $r=3$; es decir, el punto $x_{r}^{*}$ es periódico para valores de $r$ ligeramente mayores que tres. El nombre de este fenómeno dinámico quedará claro a continuación. Como habíamos mencionado antes, un punto periódico satisface que $x_{t+p}=x_{t}$, entonces buscaremos primero órbitas con período dos. Con este fin, investigamos si $x^{*}=f^{2}\left(x^{*} ; r\right)$ tiene solución. A partir de (2), obtenemos que esta ecuación es equivalente a calcular las raíces del polinomio

$$
x^{*}\left[r x^{*}+(1-r)\right]\left[r^{2} x^{*^{2}}-r(1+r) x^{*}+1+r\right]=0 .
$$

Las raíces de este polinomio están dadas por

$$
x_{0}^{*}=0, \quad x_{1}^{*}=x_{r}^{*}, \quad x_{ \pm 2}^{*}=\frac{1}{2 r}[1+r \pm \sqrt{(1+r)(r-3)}] .
$$

De esta manera, recordando que $0 \leq x_{t} \leq 1$ para todo $t \in \mathbb{N}_{0}$, observamos que si $r<3$, los únicos puntos fijos de $f^{2}$ son $x_{0}^{*}$ y $x_{r}^{*}$. Mientras que para $r>3$, el sistema $x_{t+2}=f^{2}\left(x_{t} ; r\right)$ tiene cuatro puntos fijos. Esto significa que cualquier condición inicial, con excepción de los puntos fijos, las órbitas convergen a la órbita periódica de (2) determinada por los puntos $x_{ \pm 2}^{*}$. Ahora, procediendo de la misma manera, al investigar si la condición de la bifurcación de doblamiento de período primario es satisfecha para $f^{2}$, encontramos que existen puntos periódicos de período $2^{2}$. De esta manera, observamos que el valor de $r$ en el cual ocurre una bifurcación de doblamiento de período en el intervalo $3<r \leq 4$ es equivalente a resolver la ecuación

$$
\left(f^{2}(x ; r)\right)^{\prime}=-1
$$

para $r$. Encontramos que $r=r_{1} \equiv 1+\sqrt{6}$. Esto sugiere que existe una cascada de bifurcaciones de este tipo tales que producen órbitas cuyo período aumenta en potencias de dos. En otras palabras, existen órbitas 2-periódicas para $3<r<r_{1}$; equivalentemente, órbitas $2^{2}$-periódicas aparecen cuando $r_{1}<r<r_{2}$, órbitas $2^{3}$-periódicas en el intervalo $r_{2}<r<r_{3}$ y así sucesivamente hasta que $r$ alcanza el valor $r_{\infty}=3.56994$. Cuando esto ocurre, se dice que el período de la órbita es $2^{\infty}$. Las bifurcaciones, cuando $r$ alcanza los valores $r_{2}, r_{3}, r_{4}$, etcétera, son determinados numéricamente.

A continuación, en las Figuras 1-3 se ilustra la dinámica de la ecuación logística (2) para valores característicos del parámetro Malthusiano. El cálculo es mostrado de dos maneras: 


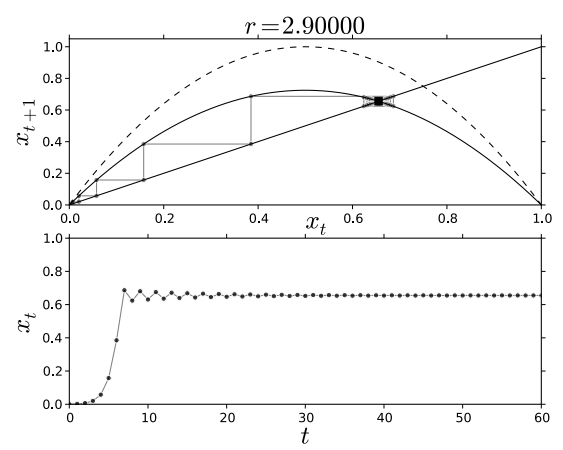

(a)

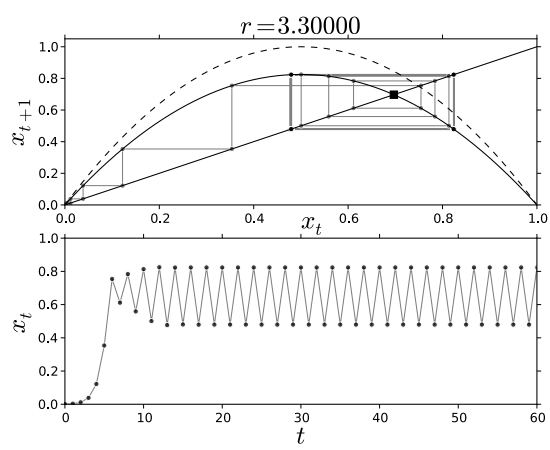

(b)

Figura 1: Soluciones de la ecuación logística para valores característicos de $r$. (a) Órbita convergente al atractor $x_{r}^{*}$; (b) órbita periódica de período $2^{1}$. En los paneles superiores, la parábola punteada corresponde a la función $f(x ; r)$ para $r=4$. El punto de intersección entre la parábola (curva sólida) y la identidad determina el punto fijo $x_{r}^{*}$, el cual es indicado por un cuadrado negro. Las bolas negras sobre la parábola graficada por una curva sólida corresponden a los máximos y mínimos locales de la evolución de $x_{t}$ para los primeros 60 valores de $x_{t}$ (paneles inferiores). La población relativa inicial es $x_{0}=0.01$ para ambos casos.

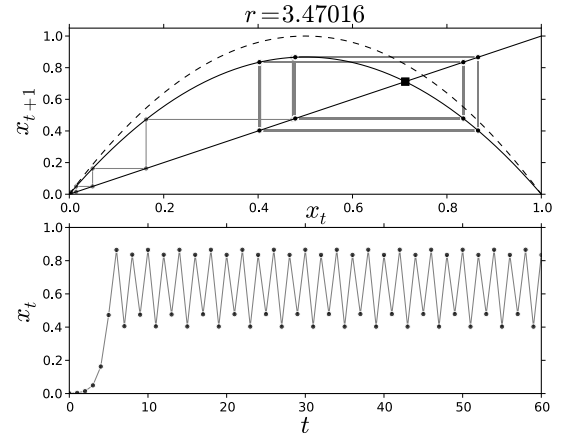

(a)

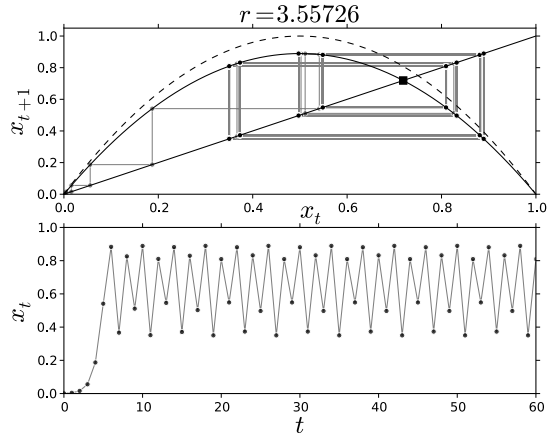

(b)

Figura 2: Soluciones de la ecuación logística para valores característicos de $r$. Órbita periódica (a) de período $2^{2}$ y (b) de período $2^{3}$. En los paneles superiores, la parábola punteada corresponde a la función $f(x ; r)$ para $r=4$. El punto de intersección entre la parábola (curva sólida) y la identidad determina el punto fijo $x_{r}^{*}$, el cual es indicado por un cuadrado negro. Las bolas negras sobre la parábola sólida corresponden a los máximos y mínimos locales de la evolución de $x_{t}$ para los primeros 60 valores de $x_{t}$ (paneles inferiores). La población relativa inicial es $x_{0}=0.01$ para ambos casos.

(i) utilizando el método de la telaraña, el estado del sistema a cada tiempo $t$ y tiempo $t+1$ está representado por una curva en el plano definido por $x_{t} \mathrm{y} x_{t+1}$. Esta órbita consiste en la colección de rectas que unen cada dos puntos consecutivos sobre la identidad y la parábola representada por las líneas sólidas. Véanse los paneles superiores de las Figuras 1-2 


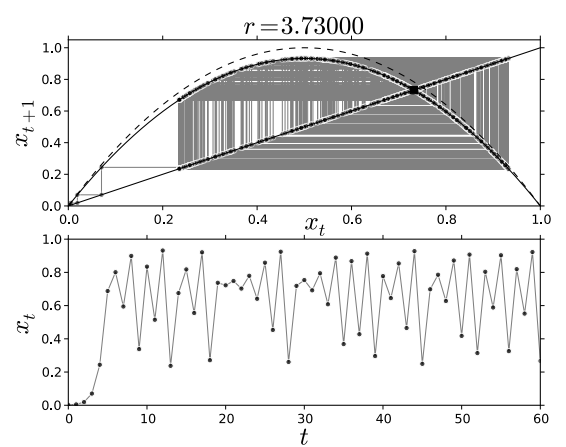

(a)

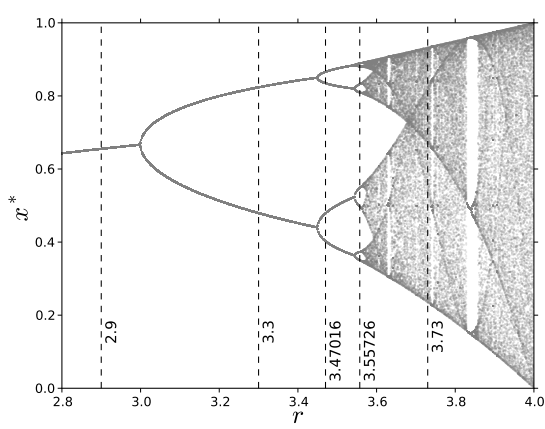

(b)

Figura 3: (a) Órbita con comportamiento caótico para la ecuación logística. En el panel superior, la parábola punteada corresponde a la función $f(x ; r)$ para $r=4$. El punto de intersección entre la parábola (curva sólida) y la identidad determina el punto fijo $x_{r}^{*}$, el cual es indicado por un cuadrado negro. Las bolas negras sobre la parábola sólida corresponden a los máximos y mínimos locales de la evolución de $x_{t}$ para los primeros 60 valores de $x_{t}$ (panel inferior). La población relativa inicial es $x_{0}=0.01$. (b) Diagrama de bifurcación al variar $r$ para $1.2 \times 10^{3}$ iteraciones; las líneas verticales punteadas representan los valores de $r$ para la dinámica en las Figuras 1 y 2 y el panel (a).

y la Figura 3(a). Por otro lado, (ii) la evolución temporal de $x_{t}$ puede verse en los paneles inferiores de las Figuras 1-2 y la Figura 3(a). Observemos que la órbita converge al atractor $x_{r}^{*}$ (Figura 1(a)), a una órbita periódica (Figuras 1(b), 2(a) y 2(b)) o una órbita sin período alguno (Figura 3(a)).

Para observar la manera en la cual el parámetro $r$ influye en el comportamiento de los puntos de equilibrio, construimos un diagrama de bifurcación, como se muestra en la Figura 3(b). En este diagrama vemos la relación que existe entre los puntos fijos $x^{*}$ de $f^{2 k}$, como función del parámetro de bifurcación $r$. Como podemos ver, las bifurcaciones de doblamiento de período inducen la aparición de nuevas ramas conforme $r$ crece. Cada punto sobre cada rama representa el valor en los cuales el sistema $x_{t+2 k}=f^{2 k}\left(x_{t} ; r\right)$ tiene puntos fijos atractores para cada $k \in \mathbb{N}_{0}$. Esto quiere decir que si

$1<r<3: x_{t}=f\left(x_{t} ; r\right)$ tiene un único atractor; la órbita es $2^{0}$-periódica;

$3<r<r_{1}: x_{t+2}=f^{2}\left(x_{t} ; r\right)$ tiene dos atractores; la órbita es $2^{1}$-periódica;

$r_{1}<r<r_{2}: x_{t+4}=f^{4}\left(x_{t} ; r\right)$ tiene cuatro atractores; la órbita es $2^{2}$-periódica;

$r_{2}<r<r_{3}: x_{t+8}=f^{8}\left(x_{t} ; r\right)$ tiene ocho atractores; la órbita es $2^{3}$-periódica.

Estas bifurcaciones aparecen cada vez más cercanas entre sí al crecer $r$. Esto significa que las órbitas doblan su período con mayor rapidez al aumentar el parámetro Malthusiano. La razón a la cual la distancia entre dos elementos consecutivos de la sucesión de bifurcaciones de este tipo decrece es universal. Esta razón se define como la primer constante de 
Feigenbaum (véase, por ejemplo, Smith, 2013):

$$
\delta=\lim _{k \rightarrow \infty} \frac{r_{k}-r_{k-1}}{r_{k+1}-r_{k}} \approx 4.6692016 \ldots
$$

Esta constante indica que conforme $r$ esté más cerca del valor crítico $r_{\infty}$, los doblamientos de período ocurren más cerca uno del otro a una razón $\delta$.

En 1964, Sharkovsky publica uno de los resultados más fascinantes de los sistemas dinámicos discretos. Este resultado, conocido como el Teorema de Sharkovsky (véase Sharkovsky, 1964), establece la existencia de órbitas $q$-periódicas, siempre y cuando $f$ sea una función continua con un punto $p$-periódico, de tal manera que $q$ preceda a $p$. Por ejemplo, la cascada de órbitas periódicas exhibidas arriba está ordenada según el llamado ordenamiento de Sharkovsky; esto es $2^{0}$ precede a $2^{1}$, el cual precede a $2^{2}$ y, a su vez, precede a $2^{3}$, etcétera. Una de las consecuencias de este teorema yace en el hecho que, si $f$ tiene una órbita $(2 k+1)$-periódica para $k \geq 1$, entonces para un algún valor $r=r_{\infty}$ existe una solución cuyo comportamiento es caótico para $r>r_{\infty}$; véase (Li y Yorke, 1975). Es importante recalcar que el ordenamiento de Sharkovsky corresponde solamente a órbitas periódicas de primera aparición. Este caso peculiar se muestra en la Figura 3(a): la población relativa no crece ni decrece periódicamente, sino que oscila sin período alguno. Como puede verse en el panel superior, la órbita cubre una infinidad de puntos sobre la parábola (curva sólida). Esto sugiere que el período es infinito.

Por otro lado, notemos que la región gris a la derecha de $r_{\infty}$ en la Figura 3(b), obtenida por medio de bifurcaciones de doblamiento de período, forma un patrón fractal.

\subsection{Entre dos dimensiones}

En el ejemplo anterior, observamos que al fijar un valor de $r$ en la región gris de la Figura 3(b), la órbita es de período inconmensurable. Es decir, los máximos y mínimos corresponden a una infinidad de valores de $x_{t}$ que yacen sobre la parábola (curva sólida) del panel superior en la Figura 3(a). La región en el diagrama de bifurcación donde esto ocurre tiene estructura fractal. En otras palabras, es un conjunto de dimensión fractal (no entera) y presenta la propiedad de autosimilitud.

Primero, entendamos que la dimensión fractal es una manera de caracterizar qué tanto un conjunto cambia a diferentes escalas respecto al espacio donde se encuentra. Con el fin de profundizar en esta noción, observemos que intuitivamente la dimensión de un objeto está relacionada con su medición a cierta escala. Es decir, si una línea de longitud unitaria aumenta por un factor de escala dos, su longitud se verá incrementada $2^{1}$ veces; si un cuadrado de área unitaria es aumentado este mismo factor, su área aumentará $2^{2}$ veces; equivalentemente, el volumen de un cubo crecerá $2^{3}$ veces. Siguiendo esta idea, podemos decir que un cambio en el factor de escala produce un aumento exponencial en el área, volumen o medida en general del objeto. En el ejemplo anterior, el exponente es un número entero y representa la dimensión del objeto, i.e. una línea es de dimensión uno, un cuadrado es de dimensión dos y un cubo es de dimensión tres. Por el contrario, los conjuntos fractales tienen 


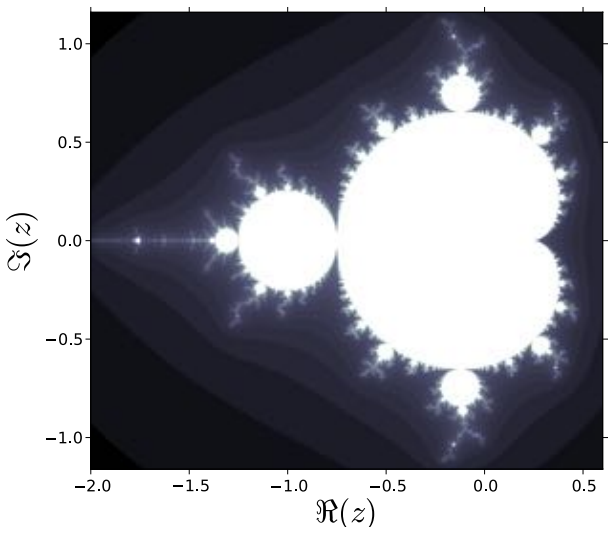

(a)

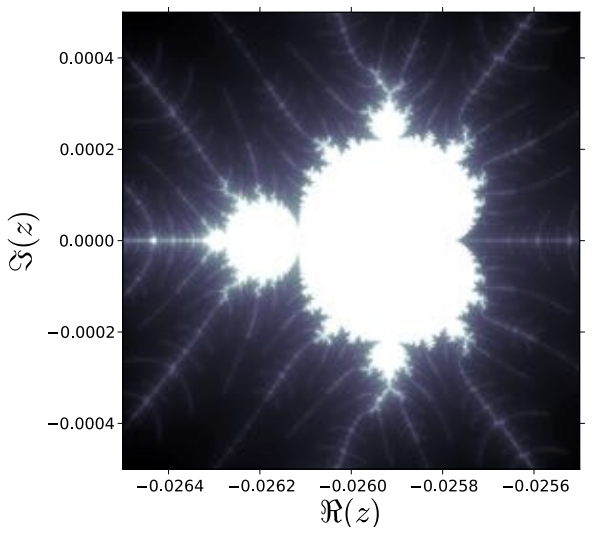

(b)

Figura 4: Propiedad de autosimilitud. (a) Conjunto de Mandelbrot en $\mathbb{C}$. (b) Aumento de escala alrededor de $z=-1.76$. Número de iteraciones: $3 \times 10^{3}$.

una dimensión que no es un número entero. Véase, por ejemplo, el libro de Peitgen, Jürgens, y Saupe (1992), para una discusión más profunda en esta dirección. Algunos ejemplos en la naturaleza con estructura fractal pueden observarse en el volumen de colonias de corales, el crecimiento de algunas bacterias, el sistema circulatorio y el sistema nervioso, entre otros. Una de las capacidades de estas estructuras fractales en la naturaleza yace en una característica asombrosa: la capacidad de capturar un balance entre longitudes y áreas o áreas y volúmenes, de tal manera que las longitudes y áreas son pequeñas en comparación con las áreas y volúmenes donde estas estructuras fractales se encuentran, respectivamente. Es decir, la estructura fractal del sistema circulatorio es tal que el área que cubre es pequeña en comparación con la longitud que se obtiene al unir cada una de las ramificaciones que lo comprenden; véase, por ejemplo, (Gabrys, Rybaczuk, y Kedzia, 2005).

Teniendo en mente lo anterior, notemos que la región en cuestión en el diagrama de bifurcación de la ecuación logística, además de contener los valores de $r$ en los cuáles es imposible determinar el valor de la población relativa en el tiempo $t+1$, también tiene regiones en forma de ventanas verticales, véase $r \approx 3.62, r \approx 3.74$ y $r \approx 3.85$, por ejemplo. En estas ventanas, la estructura del diagrama original es recuperada al hacer un acercamiento, i.e. un cambio de escala. Este fenómeno es conocido como autosimilitud.

Para ejemplificar la propiedad de autosimilitud de conjuntos con estructura fractal, tomemos en cuenta el conjunto de Mandelbrot. Este conjunto se obtiene a partir de valores de $c$ en el plano complejo, comúnmente denotado por $\mathbb{C}$, para el cual la órbita que parte de $z_{0}=0$, bajo iteraciones de $z_{n+1}=z_{n}^{2}+c$, permanece acotada. Dicho conjunto se muestra en la Figura 4(a). En particular, el conjunto de Mandelbrot tiene una estrecha relación con el diagrama de bifurcación de la ecuación logística discreta mostrado en la Figura 3(b). Sin embargo, aquí solamente nos interesaremos en la propiedad de autosimilitud. Esta propiedad puede apreciarse en la Figura 4(b); ahí se muestra una magnificación del conjunto de Mandelbrot alrededor de $z_{0}=-1.76$. Como puede verse, la figura original es recuperada. 
Los conjuntos con estructura fractal juegan un papel primordial en los sistemas que exhiben comportamiento caótico.

\section{La volubilidad en el continuo}

En el ejemplo de la ecuación logística, consideramos que el tiempo estaba dado por una colección homogénea de números enteros. Esta suposición es válida para algunos fenómenos en la naturaleza. Sin embargo, existen fenómenos donde hacer una suposición semejante puede llevar a resultados que no concuerdan con las observaciones experimentales. Por ejemplo, si quisiéramos describir la propagación de VIH debido a los contactos sexuales entre individuos de una población, considerar variables discretas es un enfoque plausible. Por el contrario, si quisiéramos entender el efecto que una hormona tiene en el proceso de curación en una planta, tendríamos que usar variables continuas, debido a que las células de la planta crecen y participan en dicho proceso en todo momento.

A continuación exploraremos dos casos donde las variables son continuas en lugar de ser discretas. Estos ejemplos exhiben comportamientos de naturaleza caótica. En el primero entenderemos las consecuencias del célebre efecto mariposa. En el segundo veremos una serie de eventos particulares que conducen a la aparición de un comportamiento caótico en la dinámica de formación de patrones desde el punto de vista de las ecuaciones de reaccióndifusión.

\subsection{El clima según Lorenz}

En 1963, Edward Lorenz dedujo una simplificación de las ecuaciones de Navier-Stokes para la convección del flujo de Bénard de un fluido (véase Lorenz, 1963). Esto con el fin de determinar las condiciones para la predicción del clima. Dicho modelo, usualmente conocido como el modelo de Lorenz, consiste en un sistema de tres ecuaciones ordinarias, que describen la convección atmosférica. Las componentes del sistema reflejan los ingredientes necesarios en la dinámica de la convección de un fluido. La razón de transferencia de calor es representada por $x$ y la temperatura de convección horizontal y vertical están dadas por y y z, respectivamente. De esta forma, el sistema de Lorenz consiste por las ecuaciones

$$
\dot{x}=\sigma(x-y), \quad \dot{y}=(\rho-z) x-y, \quad \dot{z}=x y-\beta z,
$$

donde $\sigma$ es un parámetro proporcional al cociente entre la viscosidad y la conductividad térmica del fluido (número de Prandtl), $\rho$ es proporcional a la diferencia de temperaturas en la dirección vertical (número de Rayleigh) y $\beta$, relacionado con las dimensiones físicas del sistema, es proporcional al cociente entre la altura y la longitud de la región donde el proceso de convección ocurre.

La importancia de este modelo es tal que es usado en una diversidad de fenómenos relacionados con meteorología, hidrodinámica, física de láseres, superconductividad y cinéticas bioquímicas, entre otros.

El sistema (5) tiene dos propiedades importantes: (i) todos los estados iniciales que empiezan en el eje $z$, regresan a $z$ cuando $t \rightarrow \infty$; (ii) las soluciones son idénticas ante una reflexión 


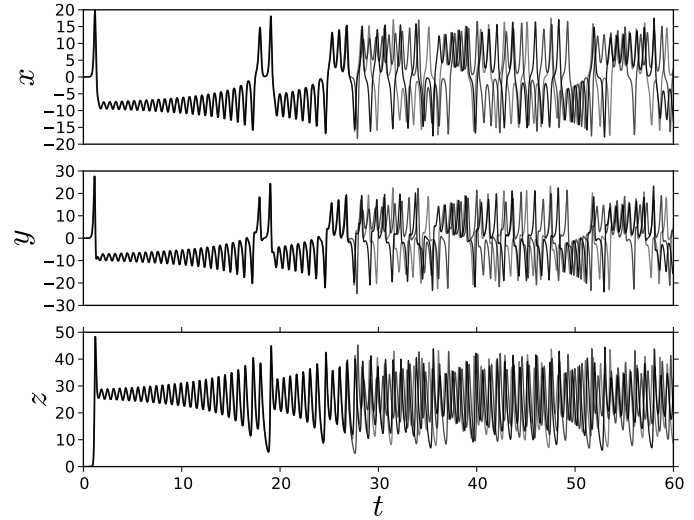

(a)

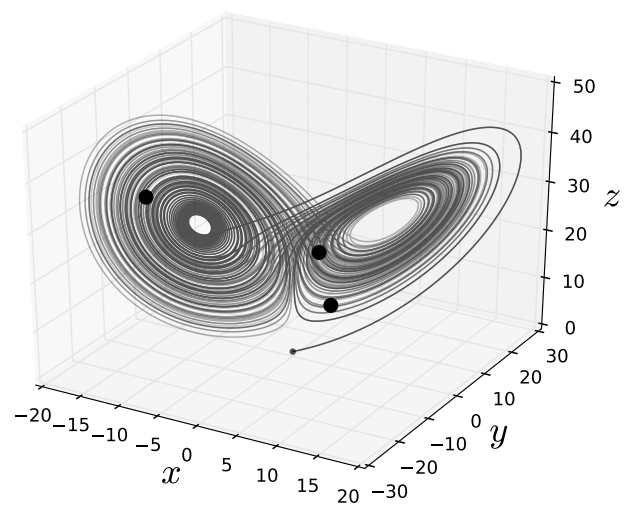

(b)

Figura 5: Sensibilidad a las condiciones iniciales. (a) La evolución de $x, y$ y $z$ respecto al tiempo y (b) la máscara de Lorenz. Los tres estados iniciales están dadas por perturbaciones aleatorias del origen de orden $10^{-4}$ para cada uno. Los estados finales, al tiempo $t=60$, están marcados por los puntos negros. Valor de los parámetros: $\sigma=10, \rho=28$ y $\beta=8 / 3$.

respecto al plano definido por $z=0$. Estas dos propiedades indican que para un estado inicial tal que la transferencia de calor y la temperatura de convección horizontal son cero, entonces la temperatura de convección del fluido regresará a la dirección vertical; si la razón de transferencia de calor o la temperatura de convección horizontal son positivas, entonces la cantidad negativa de ambas es conocida bajo la transformación $(x, y, z) \rightarrow(-x,-y, z)$, independientemente del valor de los parámetros. Esto quiere decir que hay una simetría que describe la estructura del retrato fase, donde la evolución de los estados del sistema yacen.

Resolvemos numéricamente el sistema (5). En la Figura 5(a) se muestran las evoluciones temporales de las tres componentes $x, y, z$ para tres estados iniciales distintos (curvas pintadas en negro, gris oscuro y gris claro, respectivamente). La separación entre cada estado inicial es de orden $10^{-4}$. Notemos que, aunque los estados se "acompañan" inicialmente, los estados finales difieren el uno del otro; el valor de las componentes al tiempo $t=60$ corresponden a los tres estados finales. Para visualizar esto de mejor manera, en la Figura 5(b) se muestra el retrato fase, conocido también como la máscara de Lorenz. En esta figura, los estados iniciales, representados por puntos negros pequeños, son prácticamente indistinguibles entre ellos. Por otro lado, los estados finales distan considerablemente uno del otro, indicados por los puntos negros grandes. Esto remarca la sensibilidad a las condiciones iniciales del sistema, una de las principales características del caos en sistemas deterministas. Es decir, aunque exista una variación pequeña en los estados iniciales, la distancia de estos crece exponencialmente al transcurrir el tiempo. Este es el principio básico del fenómeno conocido como el efecto mariposa. El efecto mariposa fue formulado por Lorenz en una plática que impartió en Brasil en el año de 1972 en relación a la sensibilidad a condiciones iniciales. Esta formulación 
es descrita en los siguientes términos: "Previsibilidad: ¿el aleteo de las alas de una mariposa en Brasil puede producir un tornado en Texas?"

Una característica que el sistema de Lorenz comparte con la ecuación logística radica en que la máscara de Lorenz tiene estructura fractal. Las características de ésta provienen del hecho que: (i) las órbitas (evolución temporal de los estados en el espacio fase) que lo comprenden no tienen punto de intersección alguno; (ii) sus órbitas no convergen a ningún conjunto límite, i.e. no convergen en órbitas periódicas ni a ningún punto de equilibrio; (iii) a pesar que el sistema es altamente sensible a condiciones iniciales, su forma global no depende de ello. Véase, por ejemplo, (Verhulst, 1996) para los detalles del análisis.

En resumen, además de las características anteriores, el modelo de Lorenz remarca el hecho que es imposible predecir el comportamiento del clima a largo plazo. Este modelo es fundamental para el entendimiento de las propiedades intrínsecas que conducen a comportamientos caóticos en sistemas deterministas.

\subsection{El espacio-tiempo caótico}

En 1952, Alan Turing propuso las condiciones necesarias para que la combinación de dos procesos físicos produjeran la formación de estructuras espaciales fuera del equilibrio termodinámico cuando dos sustancias químicas interactúan (véase Turing, 1952). Estos procesos son: la reacción y la difusión. Este trabajo puso en escena el estudio de las ecuaciones de reacción-difusión. Estas ecuaciones han sido una herramienta clave en el entendimiento de una diversidad de procesos morfogenéticos. Por ejemplo, la iniciación bioquímica de pelos radiculares en raíces de plantas, interacciones entre especies de animales y estudio de males cardiacos y neuronales, entre muchos otros.

En los modelos cuya columna vertebral son los sistemas de reacción-difusión, el caos espaciotemporal ha sido estudiado en las últimas décadas. Aquí solamente presentamos algunos de los resultados publicados recientemente en (Aragón, Barrio, Woolley, Baker, y Maini, 2012). En este trabajo, R. Barrio, C. Varea, J. L. Aragón y P. Maini señalan una sucesión de eventos característicos que conducen al caos espacio-temporal en el sistema de reaccióndifusión denominado $B V A M$, por las siglas de los apellidos de los autores.

El sistema BVAM está compuesto por dos ecuaciones diferenciales parciales no lineales acopladas. En términos simples, el sistema describe de forma general la interacción dinámica de dos concentraciones de sustancias químicas que se difunden en un medio homogéneo. Supongamos que estos dos procesos ocurren en una dimensión espacial, es decir, las sustancias "viven" en el intervalo $[0, L]$. Las variables de estado, $u(t, x)$ y $v(t, x)$ representan la concentración de cada sustancia al tiempo $t$ en la posición $x$. La razón de cambio temporal instantánea de $u$ y $v$ es directamente proporcional a la difusión y la reacción de las sustancias, donde el término de difusión proviene de la aproximación macroscópica del movimiento Browniano de las partículas que comprenden cada una de las sustancias; este término está dado por la segunda derivada con respecto a $x$. Por otro lado, la Ley de acción de masas determina la razón de cambio instantáneo del producto de una reacción (bio)química es proporcional al producto de las concentraciones. Como elemento clave, se supone que las concentraciones $u$ 
y $v$ están aisladas y solamente interactúan entre ellas. Reuniendo estos elementos, se obtiene el sistema BVAM,

$$
u_{t}=D u_{x x}+\eta\left(u+a v-c u v-u v^{2}\right), \quad v_{t}=v_{x x}+\eta\left(h u+b v+c u v+u v^{2}\right),
$$

donde $D$ es el cociente entre los coeficientes de difusión de ambas concentraciones (la rapidez de difusión relativa ), $a, b, h$ y $c$ son los parámetros cinéticos y $\eta$ es un parámetro que indica la intensidad de la reacción. El sistema (6) no modela un fenómeno en particular, sino que captura de manera general los ingredientes relevantes de una interacción química bajo un proceso de difusión. El tamaño del dominio está dado por $L=10$ y la interacción es aislada, lo cual indica que el flujo de ambas concentraciones en los extremos del dominio es nulo, i.e. $u_{x}=v_{x}=0$ cuando $x=0$ y $x=L$, respectivamente.

En general, los sistemas de reacción-difusión exhiben una colección de familias de soluciones con características específicas. Si un conjunto de transiciones, o bifurcaciones, entre cada una de estas familias ocurre en un orden específico, es posible obtener comportamiento caótico. $\mathrm{Al}$ variar el parámetro $c$, un orden particular de bifurcaciones fue descubierto en el sistema BVAM. Aunque no particularmente inherente a este sistema, esta sucesión de bifurcaciones es conocido como la ruta al caos de tipo Ruelle-Takens-Newhouse.

$\mathrm{Al}$ decrecer negativamente $c$, esta ruta sucede de la siguiente manera: a partir de una familia de soluciones estacionarias en el tiempo, la primer bifurcación que ocurre es tal que las soluciones del sistema oscilan en el tiempo con un cierto período que depende de los parámetros de los términos cinéticos; cuando una bifurcación de este tipo ocurre, se le conoce como bifurcación de Hopf. Enseguida, una bifurcación de doblamiento de período aparece. Al igual que en el ejemplo de la ecuación logística, las soluciones oscilan en el tiempo con dos períodos distintos, los cuales son conmensurables, i.e. el cociente de los períodos de oscilación es un número racional. Finalmente, al continuar variando $c$ negativamente, una tercera bifurcación ocurre, la cual produce una tercera oscilación en el tiempo. El período de esta tercera oscilación es inconmensurable con los otros dos períodos; esta bifurcación es llamada bifurcación tórica, también conocida como bifurcación secundaria de Hopf. Después de este evento, un comportamiento caótico emerge. En otras palabras, la sucesión de eventos que se espera observar es cualitativamente similar a la que vimos en el modelo logístico. Sin embargo, existe una diferencia fundamental: la aparición de una oscilación cuyo período no es posible obtener a partir de las oscilaciones previas. Véase, por ejemplo, (Wiggins, 2003) para una discusión detallada de cada una de estas bifurcaciones.

En la Figura 6 se muestran tres valores distintos del parámetro $c$ para el cual ocurren los principales ingredientes de la ruta al caos descrita en el párrafo anterior. En la columna de la izquierda vemos el retrato fase de $u$ y $v$ en el punto $x=4$, los cuadrados y círculos en blanco representan los estados inicial y final, respectivamente. En los paneles superiores de la columna derecha se observa la evolución temporal de $u$ en $x=4$ para el intervalo de tiempo ahí indicado. En los paneles inferiores de la misma columna, se muestra la evolución espaciotemporal de $u$. En las Figuras 6(a)-(b) se observa la evolución de un estado estacionario cuya estructura espacial es heterogénea; en particular, la emergencia de estado proviene de una bifurcación de Turing (véase Murray, 2002) para los detalles teóricos. Enseguida, en las 


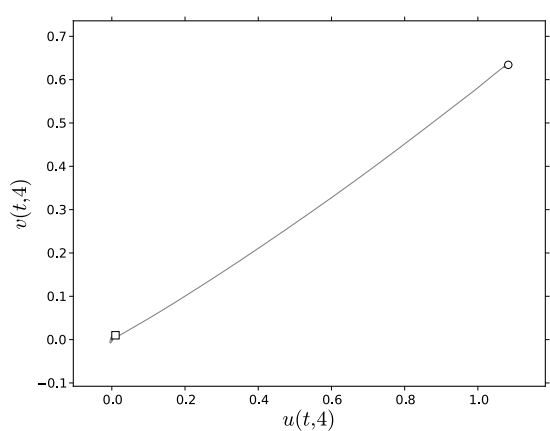

(a)

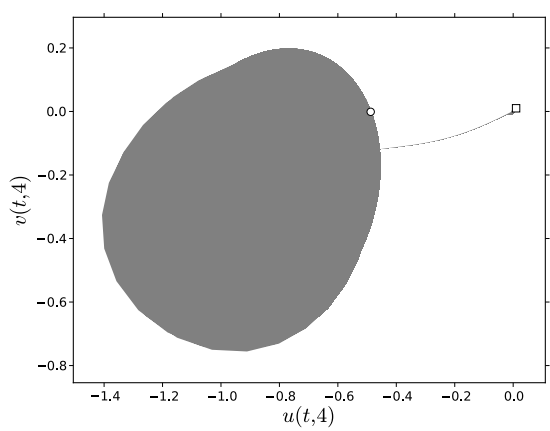

(c)

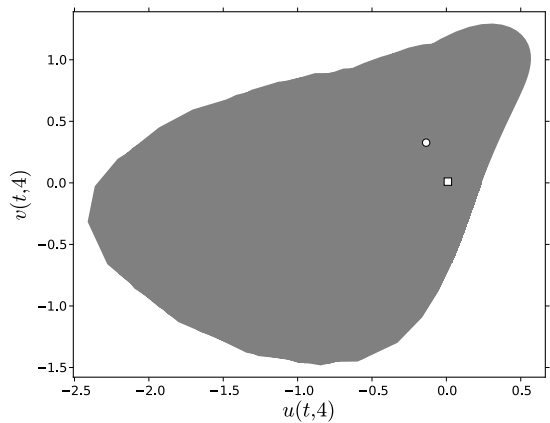

(e)

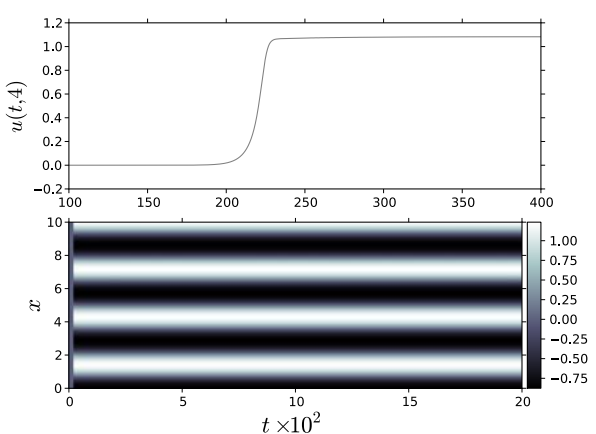

(b)

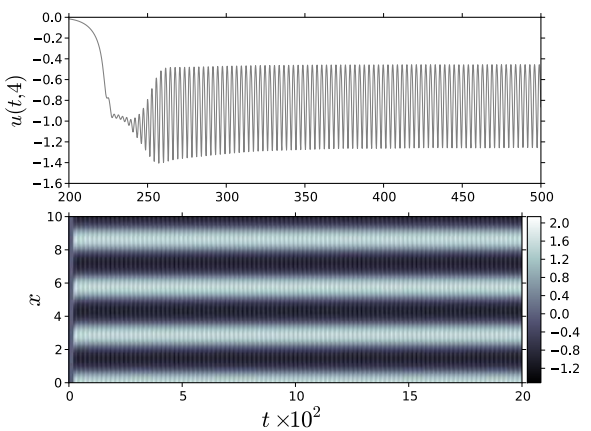

(d)

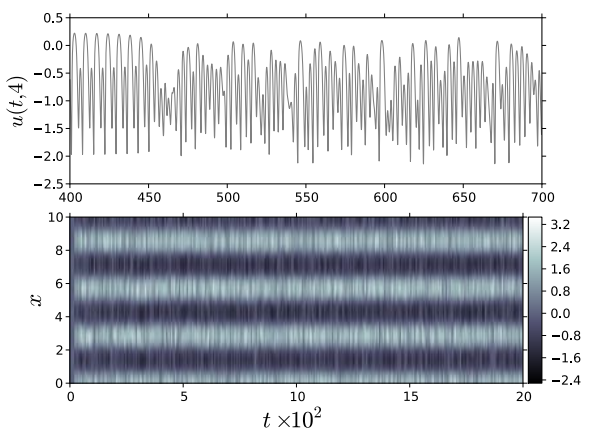

(f)

Figura 6: Ruta al caos de tipo Ruelle-Takens-Newhouse en el sistema de reacción-difusión BVAM. (a)-(c)-(e) Espacio fase de $u$ y $v$ evaluadas en el punto $x=4$; las condiciones inicial y final están indicadas por un cuadrado y un círculo blancos respectivamente. (b)-(d)-(f) Muestra de la evolución temporal de $u$ en $x=4$ (paneles superiores) y evolución espacio-temporal (paneles inferiores) de $u$ en todo el intervalo. Valor de los parámetros: $a=-1, b=-3 / 2$, $h=3, \eta=1, D=0.08$ y (a)-(b) $c=-0.6$, (c)-(d) $c=-1$, (e)-(f) $c=-1.5$; condiciones iniciales: $u_{0}=v_{0}=0.01$.

Figuras 6(c)-(d) se muestra una oscilación que predomina en el transcurso del tiempo. Es decir, después de un intervalo de tiempo transitorio, es posible observar oscilaciones con un 
período determinado. Finalmente, en las figuras 6(e)-(f), se muestra el caos espacio-temporal que este sistema exhibe. Aunque la evolución de estados, en particular en el punto $x=4$, es acotado, el período es infinito. Además, al construir una sección de Poincaré, la cual consiste en trazar una línea con cualquier pendiente en el plano fase. La intersección de dicha línea con la órbita determinan una sucesión de puntos; estos puntos forman un conjunto que, para el caso que se muestra en la Figura 6(e), tiene estructura fractal.

El escenario caótico que el sistema BVAM presenta parece tener los ingredientes dinámicos necesarios para la descripción de una falla cardiaca. Sin embargo, por el momento, este sistema carece de una interpretación fisiológica adecuada.

\section{Comentarios finales}

Llegados a este punto, aterricemos las ideas expuestas en este artículo. Primero, el corte descrito al final del ejemplo anterior recibe el nombre de sección de Poincaré y el método que reúne la colección de puntos sobre dicha sección es conocida como la aplicación de Poincaré. Este método permite el estudio de propiedades dinámicas que ocurren en los sistemas dinámicos no lineales; por ejemplo, en la determinación de la naturaleza de una órbita, la cual puede ser atractora o repulsora. De esta manera, no solamente se simplifica el análisis en los espacios fase, sino que permite describir comportamientos como los que aquí se presentan. De esta manera, el conjunto formado por una aplicación de Poincaré para el sistema de Lorenz y el sistema BVAM en los respectivos espacios fase tienen estructura fractal. La cual es una característica que el diagrama de bifurcación de la ecuación logística comparte.

Hasta nuestros días, no existe una definición universal de caos; sin embargo, la comunidad científica en el mundo concuerda en que los sistemas caóticos tienen como ingredientes esenciales: (i) la ocurrencia de órbitas oscilantes que no convergen en puntos fijos o de equilibrio $\mathrm{u}$ órbitas periódicas cuando $t \rightarrow \infty$; (ii) la sensibilidad a condiciones iniciales y la aparición de estructuras fractales; (iii) son deterministas, es decir, su dinámica no tiene origen en un comportamiento aleatorio, sino que proviene de su estructura dinámica no lineal. Como vimos en los ejemplos, las estructuras fractales están relacionadas con propiedades intrínsecas al sistema y, por tanto, al fenómeno en estudio. En general, en sistemas que exhiben comportamientos caóticos, los puntos de equilibrio que resultan ser atractores y que además tienen propiedades como el mostrado en el origen del sistema de Lorenz en la Figura 5 y las Figuras 6(e)-(f) para el sistema BVAM, se les conoce como atractores extraños. En otras palabras, los atractores extraños pueden entenderse de manera simplificada como atractores relacionada a una estructura fractal. sin embargo, es importante señalar que no todo atractor extraño está relacionado con un comportamiento caótico y viceversa (véase Ivancevic y Ivancevic, 2008; Strogatz, 2015, porjemplo).

Para un recorrido más profundo al mundo de los sistemas caóticos, en el libro escrito por Alligood, Sauer, y Yorke (1996) se expone una introducción básica, aunque detallada, de los sistemas dinámicos no lineales que exhiben caos. Desde el punto de vista de la Física, una muy interesante inmersión al mundo de la complejidad no lineal, donde el caos es ca- 
racterística primordial, puede encontrarse en el libro de Ivancevic y Ivancevic (2008) y más recientemente en la última edición del libro de Strogatz (2015). Por otro lado, los libros de Wiggins (2003) y Gleick (1987) son referencias esenciales en el estudio de los sistemas dinámicos $\mathrm{y}$, particularmente, el caos.

\section{Agradecimientos}

Agradezco cálidamente al Comité Editorial por la invitación a participar en la revista Sahuarus con el presente ensayo. Asimismo, agradezco a los revisores del mismo, cuyos comentarios y sugerencias mejoraron considerablemente la exposición de este manuscrito.

\section{Referencias}

Alligood, K., Sauer, T., y Yorke, J. (1996). Chaos: An Introduction to Dynamical Systems. NewYork: Springer-Verlag.

Aragón, J., Barrio, R., Woolley, T., Baker, R., y Maini, P. (2012). Nonlinear Effects on Turing Patterns: Time Oscillations and Chaos. Phys. Rev. E, 86(2), 026201.

Gabrys, E., Rybaczuk, M., y Kedzia, A. (2005). Fractal models of circulatory system. Symmetrical and asymmetrical approach comparison. Chaos, Solitons and Fractals, 24, pp. 707-715.

Gleick, J. (1987). Chaos: Making a New Science. New York: Viking Penguin Inc.

Ivancevic, V., y Ivancevic, T. (2008). Complex Nonlinearity. New York: Springer-Verlag.

Laplace, P. (1902). A Philosophical Essay on Probabilities (traducido del francés, $6^{\circ}$ ed.; F. Truscott y F. Emory, Eds.). London: John Wiley \& sons.

Li, T.-Y., y Yorke, A. (1975). Period Three Implies Chaos. The American Mathematical Monthly, 82(10), pp. 985-992.

Lorenz, E. (1963). Deterministic Nonperiodic Flow. J. Atmos. Sci., 20, pp. 130-141.

May, R. (1976). Simple Mathematical Models With Very Complicated Dynamics. Nature, 261, pp. 459-467.

Murray, J. (2002). Mathematical Biology II: Spatial Models and Biomedical Applications (3 ed.). New York: Springer-Verlag.

Peitgen, H., Jürgens, S., y Saupe, D. (1992). Chaos and Fractals. New York: Springer-Verlag.

Pickover, C. A. (2009). The Math Book: From Pythogoras to the $57^{\text {th }}$ Dimension, 250 Milestone in the History of Mathematics. New York: Sterling.

Poincaré, H. (1899). Les méthodes nouvelles de la mécanique celeste. Gauthier-Villars et fils.

Robinson, C. (1999). Dynamical Systems; Stability, Symbolic Dynamics and Chaos. US: CRC Press LLC.

Schiff, J. L. (2008). Cellular Automata: A Discrete View of the World. Wiley-Iterscience.

Sharkovsky, A. (1964). Coexistence of cycles of a continuous map of a line into itself. Uk. Math. J., 16, pp. 61-71.

Smith, R. (2013). Period doubling, information entropy, and estimates for Feigenbaum's constants. Int. J. Bifurcation Chaos, 23(11), 1350190.

Strogatz, S. H. (2015). Nonlinear Dynamics and Chaos. Whit Applications to Physics, Biology, Chemistry, and Engineering (2nd ed.). New York: Westview Press.

Turing, A. M. (1952). The chemical basis of morphogenesis. Phil. Trans. R. Soc. Lond. B, 237(641), pp. $37-72$. 
Verhulst, F. (1996). Nonlinear Differential Equations and Dynamical Systems. New York: SpringerVerlag.

Wiggins, S. (2003). Introduction to Applied Nonlinear Dynamical Systems and Chaos. New York: Springer-Verlag. 